\title{
UNA VISIÓN DE EXÁMENES NACIONALES PARA EL NUTRICIONISTA: UNA PERSPECTIVA FUTURA PARA CHILE
}

\section{AN OVERVIEW OF NATIONAL EXAMINATIONS FOR NUTRITIONISTS: A FUTURE PERSPECTIVE FOR CHILE}

\author{
María Angélica González S. \\ Departamento de Nutrición y Salud Pública, \\ Facultad de Ciencias de la Salud y de los Alimentos, Universidad del Bío-Bío, Chile.
}

\begin{abstract}
There are currently 68 institutions in Chile offering a Nutrition and Dietetics program, but only six of these are accredited. It is therefore essential that a national examination be administered to measure the minimum competencies for entry-level practitioners. Examinations of four countries were studied. The purpose of the examination in Colombia is to investigate the cognitive skills profile, identify relevant information, interpret situations, discuss decisions, and propose professional alternative actions in each of the nutrition components. In Mexico, it identifies if the graduates have the necessary knowledge and skills to effectively enter professional practice. The examination in Canada has been designed to confirm a practice based on knowledge and application of critical thinking skills. It measures the minimum competency level that the dietitian should possess, and its sole purpose is to distinguish between competent and incompetent entry-level practitioners. The objective of the examination in the United States is to protect the public's health by determining an individual's competence to practice, and is a computerized interactive test. It is concluded that a national examination to practice as a nutritionist in Chile should be applied as soon as possible.
\end{abstract}

Key words: National examination, nutrition, competencies, knowledge and skills.

Este trabajo fue recibido el 2 de Agosto de 2011 y aceptado para ser publicado el 12 de Diciembre de 2011.

\section{INTRODUCCIÓN}

Uno de los ejes fundamentales para el avance del Modelo de Atención en Salud es aplicar políticas de calidad sobre el desempeño del personal, el cual es un factor esencial en la mejoría de la calidad de vida y de salud de la sociedad.

De acuerdo al Consejo Nacional de Educación Superior dependiente del MINEDUC en Chile en el año 2011 hay 68 opciones que ofrecen la carrera de Nutrición y Dietética o Licenciatura en Nutrición y Dietética (1), 27 Universidades otorgando título de Nutricionista, 4 Institutos profesionales a lo largo del país, repartidos entre instituciones públicas y privadas; sólo 6 de ellas están acreditadas. Los curriculum formativos no se encuentran homologados, ni existe una estandarización del perfil de los docentes, que aseguren la calidad de la educación (2). Por ello, presentan desiguales programas de estudio, diferente capacitación de los profesores que imparten las clases e incluso con distinta duración (8 a 10 semestres). Todas ofrecen el título profesional de Nutricionista y algunas otorgan además el grado de licenciado en Nutrición y Dietética.

La matrícula de alumnos nuevos al año 2010 alcanzó a 2939 alumnos (con un aumento del $285 \%$ respecto al año 2005). De los centros formadores, las universidades pertenecientes al Consejo de Rectores de las Universidades Chilenas ${ }^{1}$ representan el $15,8 \%$ de la matrícula de primer año, los de universidades privadas autónomas el $72,7 \%$ y los de institutos profesionales no universitarios el $11,5 \%$ (3). El total de alumnos que estudiaban nutrición y Dietética el 2010 era de 8.868 estudiantes (4).

El modelo de educación vigente en Chile incentiva la apertura de nuevos lugares de formación superior, universitarios y/o institutos profesionales, sin velar por 
la calidad de la enseñanza que en ellas se imparte, lo que debiera constituir un motivo prioritario del Ministerio de Educación, si se espera brindar a la población, una atención óptima y ética de Salud, brindada por profesionales de salud con formación de nivel universitario (5).

De acuerdo a lo anterior, los colegios de profesionales de la salud se plantean algunas preguntas, tales como ¿qué requisitos debiera exigir el MINSAL, como garante de la calidad de salud de las personas, con respecto a la formación del capital humano?, el cual es uno de los principales empleadores de nutricionistas, ¿la calidad en la formación es igual en todos los nutricionista no importando la institución en la cual se formó?

Con todo lo expuesto, se desprende que resulta indispensable aplicar un examen que mida las competencias mínimas para entrar a ejercer la profesión. En el terreno de la educación, como en todas las actividades humanas, la evaluación es el proceso que permite valorar los aciertos, reconocer las fallas y detectar potencialidades. Contar con información válida y confiable garantiza tomar decisiones acertadas.

En Chile el examen nacional para los nutricionista que ingresan a la profesión no existe, sin embargo hay varios países que ya lo han implementado, como Colombia, Canadá, Estados Unidos y México. La experiencia de estos países, así como su aplicación es variada, por ello a continuación se expone la experiencia de 4 países, la que podría servir de base para iniciar su aplicación en Chile.

\section{COLOMBIA}

Los Exámenes de Calidad de la Educación Superior -ECAES- (6), son herramientas de estado, constituyen una modalidad de evaluación externa (Evaluación externa, es aquella que se realiza fuera de la institución educativa, en este caso es la desarrollada por el Estado, la cual complementa y enriquece la evaluación interna), se aplica a los estudiantes próximos a egresar de los programas de pregrado de educación superior. Los ECAES tienen carácter obligatorio para dichos estudiantes y, adicionalmente, pueden presentarlo voluntariamente aquellas personas que deseen autoevaluarse en cada programa del nivel de formación universitaria. En el proceso de diseño y construcción participan la Asociación Colombiana de Facultades de Nutrición y Dietética y La Asociación Colombiana de Dietistas y Nutricionistas.

El proceso de desarrollo del ECAES para Nutrición y Dietética orientado por la Universidad Nacional de Colombia se ha planteado y trabajado desde una estructura de participación con y desde las Facultades y Programas Universitarios de Formación Profesional del país ${ }^{2}$, Tienen como uno de sus objetivos fundamentales comprobar el grado de desarrollo de las competencias de los estudiantes próximos a culminar los programas académicos de pregrado que ofrecen las instituciones de educación superior, hayan aprobado por lo menos el $75 \%$ de los créditos académicos del programa correspondiente o que tengan previsto graduarse en el año siguiente.

Los lineamientos para la formación del nutricionista dietista en Colombia, se basaron en las recomendaciones de la primera y segunda Conferencias de Adiestramiento del Nutricionista Dietista en América Latina, con planes de estudio que comprendían ocho semestres teóricoprácticos y los dos últimos eminentemente prácticos.

Los ECAES apuntan a evaluar aquellas áreas y componentes fundamentales del saber que identifican la formación de cada profesión, disciplina u ocupación. Así los componentes básicos que, en consecuencia, sarán parte del examen de Nutrición y Dietética son:

Nutrición Normal: Estudio de los fundamentos de la alimentación y nutrición, comprende la descripción y análisis de los nutrientes; sus procesos de ingestión, digestión, absorción, metabolismo y excreción; requerimientos y recomendaciones de energía y nutrientes para individuos y grupos sanos durante el proceso vital humano; conceptos y aplicación de la evaluación del estado nutricional.

Nutrición Clínica: Estudio del proceso de atención nutricional de individuos enfermos que incluye la evaluación y diagnóstico nutricional; determinación de los requerimientos y recomendaciones nutricionales; formulación, seguimiento y ajuste del plan nutricional, en las diferentes etapas del proceso vital humano. Comprende 3 subcomponentes: General, Materno-infantil y del adulto.

Alimentos y Servicios de Alimentación. Comprende 2 subcomponentes:

1. Ciencia de los alimentos: Estudio integral del alimento como eje fundamental en los procesos de alimentación y nutrición del ser humano desde su composición, como vehículo de nutrientes y otros compuestos; procesos de transformación que favorezcan su biodisponibilidad e inocuidad; y otros aspectos culturales, y sociales para el logro de una alimentación saludable.

2. Administración de servicios de alimentación: Estudio y caracterización del cliente, el mercado y la organización y funcionamiento de servicios de alimentación para colectivos sanos y/o enfermos, garantizando la calidad nutricional y dietética con el fin de promover la salud, prevenir la enfermedad y lograr la calidad integral. 
Nutrición en Salud Pública: Estudio de los procesos de alimentación y nutrición en colectivos humanos: procesos sociales, culturales, económicos, políticos y éticos que inciden en el bienestar nutricional de una población. Procesos para la generación de políticas, diseño y ejecución de planes, programas y proyectos de alimentación y nutrición; seguimiento, control y evaluación de intervenciones alimentarias y nutricionales.

\section{Competencias a evaluar}

Se evidencia en dimensiones de las competencias comunicativas: interpretativa, argumentativa y propositiva.

Interpretar implica: relacionar y confrontar significados, justificar una afirmación, explicar los por qué de una proposición, demostrar la articulación de conceptos, organizar premisas y relaciones causales para sustentar una conclusión. Requiere habilidades de comprensión de la información tales como: describir, discriminar, medir, representar, seleccionar, ordenar, especificar, entender, remplazar, relacionar, comparar, clasificar, agrupar, o analizar. Para ello el estudiante debe apropiarse de conocimientos básicos que le permitan reconocer problemáticas específicas.

Argumentar es profundizar, asumiendo un punto de vista coherente y riguroso ante una temática o problemática alimentaria y/o nutricional, a través de conceptualizaciones, procedimientos y actitudes. Se refiere al hecho de explicar relaciones e hipótesis formuladas en cada área en particular. Requiere habilidades de análisis e integración de la información tales como: resolver, contrastar, confrontar, sintetizar, integrar, inferir, justificar, ejemplificar, demostrar o explicar.

Proponer implica asumir una postura constructiva y creativa, plantear opciones o alternativas ante una problemática alimentaria y/o nutricional, presente en una situación determinada. Requiere habilidades de aplicación y valoración de la información: diseñar, planear, ejecutar, transferir, solucionar, verificar, desarrollar, aplicar, comprobar, resolver, extrapolar, producir, crear, inventar, sugerir, conjeturar, deducir, formular, decidir, juzgar, concluir, cuestionar o predecir.

Con esta prueba se tiene el propósito de indagar por el perfil de competencias cognoscitivas de los evaluados, al identificar información relevante, interpretar situaciones, argumentar decisiones y proponer alternativas de acción profesional en cada una de los componentes de la Nutrición.

El examen se aplica desde el año 2009 en dos sesiones. La primera sesión de cuatro horas y media, y la segunda de cuatro horas. La estructura del examen se muestra en la tabla 1.

\section{MÉXICO}

El Centro Nacional de Evaluación para la Educación Superior, A.C. (CENEVAL) (7) es una asociación civil que ofrece, desde 1994, servicios de evaluación a cientos de escuelas, universidades, empresas, autoridades educativas, organizaciones de profesionales y de otras instancias particulares y gubernamentales. Su actividad principal es el diseño y la aplicación de instrumentos de evaluación. Su misión consiste en proveer información confiable sobre los aprendizajes que logran los estudiantes de distintos niveles educativos.

El propósito del Examen General para el Egreso de la Licenciatura Nutrición (EGEL-NUTRI) es identificar si los egresados de la licenciatura en Nutrición cuentan con los conocimientos y habilidades necesarios para iniciarse eficazmente en el ejercicio de la profesión. La información que ofrece el examen puede ser usada por el estudiante que rindió el examen, las Instituciones de Educación superior (IES), los empleadores y a la

\section{TABLA 1}

Componentes

Nutrición normal

Nutrición clínica

Alimentación y servicios de alimentación

Nutrición en Salud Pública

Comprensión lectora

Inglés

Número total de preguntas
Número de preguntas por componente

$$
\begin{gathered}
44 \\
38 \\
40 \\
38 \\
15 \\
45 \\
220
\end{gathered}
$$

Tipos de preguntas: Selección múltiple con única respuesta.

Este tipo de preguntas consta de un enunciado o problema y cuatro opciones de respuesta de las cuales sólo una es correcta. 
sociedad.

Permite a la persona que rinde el examen:

- Conocer el resultado de su formación en relación con un estándar de alcance nacional mediante la aplicación de un examen confiable y válido, probado con egresados de instituciones de educación superior (IES) de todo el país.

- Conocer el resultado de la evaluación en cada área del examen, por lo que puede ubicar aquéllas donde tiene un buen desempeño, así como aquéllas en las que presenta debilidades.

- Beneficiarse curricularmente al contar con un elemento adicional para integrarse al mercado laboral. A las Instituciones de Educación superior (IES) les permite:

- Incorporar el EGEL-NUTRI como un medio para evaluar y comparar el rendimiento de sus egresados con un parámetro nacional, además del uso del instrumento como una opción para titularse.

- Contar con elementos de juicios válidos y confiables que apoyen los procesos de planeación y evaluación curricular que les permita emprender acciones capaces de mejorar la formación académica de sus egresados, adecuando planes y programas de estudio.

- Aportar información a los principales agentes educativos (autoridades, organismos acreditadores, profesores y estudiantes) acerca del estado que guardan sus egresados, respecto de los conocimientos y habilidades considerados necesarios para integrarse al campo laboral.

A los empleadores y a la sociedad les permite:

- Conocer con mayor precisión el perfil de los candidatos por contratar y de los que se inician en el ejercicio profesional, mediante elementos válidos, confiables y objetivos de juicio, para contar con personal de calidad profesional, acorde con las necesidades nacionales.

Está dirigido a los egresados de la licenciatura en Nutrición, que hayan cubierto el $100 \%$ de los créditos, estén o no titulados, y en su caso a estudiantes que cursan el último semestre de la carrera, siempre y cuando la institución formadora así lo solicite.

Con el propósito de asegurar pertinencia y validez en

\section{TABLA 2}

\section{Atributo}

Definición

Especializado

para la carrera profesional de nutrición

De alcance nacional

Estandarizado

Criterial

De máximo esfuerzo

Objetiva

Sensible a la instrucción
Se orienta a evaluar los conocimientos y habilidades que son específicos de la formación profesional del licenciado en nutrición. No incluye conocimientos y habilidades profesionales genéricos o transversales.

Considera los aspectos de formación que son esenciales en la licenciatura en Nutrición para iniciarse en el ejercicio de la profesión en el país. No está referido a un currículo en particular.

Cuenta con reglas fijas de diseño, elaboración, aplicación y calificación.

Los resultados de cada egresado que rinde el examen se comparan contra un patrón o estándar de desempeño preestablecido por el Consejo Técnico del examen.

Permite establecer el nivel de rendimiento del egresado, sobre la base de que éste hace su mejor esfuerzo al resolver las preguntas de la prueba.

Tiene criterios de calificación unívocos y precisos, lo cual permite su automatización.

Evalúa resultados de aprendizaje del programa de formación profesional de la licenciatura en Nutrición, los cuales son una consecuencia de la experiencia educativa institucionalmente organizada. 
los instrumentos de evaluación, el CENEVAL se apoya en Consejos Técnicos integrados por expertos en las áreas que conforman la profesión, los cuales pueden representar a diferentes instituciones educativas, colegios o asociaciones de profesionales, instancias empleadoras de los sectores público y privado y de carácter independiente. Estos Consejos Técnicos funcionan de acuerdo a un reglamento y se renuevan periódicamente.

El contenido del EGEL-NUTRI es el resultado de un complejo proceso metodológico, técnico y de construcción de consensos en el Consejo Técnico y en sus Comités Académicos de apoyo en torno a:

I. La definición de principales funciones o ámbitos de acción del profesional

II. La identificación de las diversas actividades que se relacionan con cada ámbito

III. La selección de las tareas indispensables para el desarrollo de cada actividad

IV. Los conocimientos y habilidades requeridos para esas tareas profesionales
V. La inclusión de estos conocimientos y habilidades en los planes y programas de estudio vigentes de la licenciatura en Nutrición

Todo esto tiene como referente fundamental la opinión de centenares de profesionales activos en el campo de la Nutrición, formados con planes de estudios diversos y en diferentes instituciones; en una encuesta nacional, ellos aportaron su punto de vista respecto a:

a. Las tareas profesionales que se realizan con mayor frecuencia

b. El nivel de importancia de esas tareas en el ejercicio de su profesión

c. El estudio o no, durante la licenciatura, de los conocimientos y habilidades que son necesarios en esas tareas.

\section{Características del EGEL-NUTRI}

Es un instrumento de evaluación que puede describirse como un examen con los siguientes atributos (tabla 2)

\begin{tabular}{|c|c|c|c|c|c|}
\hline \multicolumn{5}{|c|}{ ESTRUCTURA DEL EGEL-NUTRICIÓN } & \\
\hline & \multirow[t]{2}{*}{ Área/Subárea } & \multirow[t]{2}{*}{$\begin{array}{c}\% \text { en } \\
\text { el examen }\end{array}$} & \multirow[t]{2}{*}{$\begin{array}{c}\text { Núm. } \\
\text { de preguntas }\end{array}$} & \multicolumn{2}{|c|}{$\begin{array}{l}\text { Distribución de } \\
\text { preguntas por sesión }\end{array}$} \\
\hline & & & & $1^{\mathrm{a}}$ & $2^{\mathrm{a}}$ \\
\hline $\mathbf{A}$ & Atención clínica nutricional & 50.61 & 82 & 82 & \\
\hline 1 . & Evaluación del estado de nutrición & 20.37 & 33 & 33 & \\
\hline 2 . & Tratamiento nutricional individualizado & 16.66 & 27 & 27 & \\
\hline 3. & Orientación alimentaria individualizada & 8.64 & 14 & 14 & \\
\hline 4. & Administración del servicio de nutrición clínica & 4.93 & 8 & 8 & \\
\hline $\mathbf{B}$ & Atención nutricional a grupos de individuos & 16.04 & 26 & & 26 \\
\hline 1 . & Evaluación de la situación alimentaria y nutricional & 6.17 & 10 & & 10 \\
\hline 2 . & Desarrollo de intervenciones nutricionales & 7.40 & 12 & & 12 \\
\hline 3. & Investigación & 2.47 & 4 & & 4 \\
\hline $\mathbf{C}$ & Administración de los servicios de alimentos & 33.33 & 54 & & 54 \\
\hline 1. & Manejo de servicios de alimentos & 11.11 & 18 & & 18 \\
\hline 2. & Normatividad para el control sanitario & 7.40 & 12 & & 12 \\
\hline 3. & Diseño de planes alimentarios y menús & 8.02 & 13 & & 13 \\
\hline 4. & Orientación alimentaria & 6.80 & 11 & & 11 \\
\hline & Total & 100 & 162 & 82 & 80 \\
\hline & *Reactivos piloto & & & & \\
\hline & Total & & & & \\
\hline
\end{tabular}


El examen está organizado en áreas, sub áreas y aspectos por evaluar. Las áreas corresponden a los ámbitos profesionales en los que actualmente se organiza la labor del licenciado en Nutrición. Las sub áreas comprenden las principales actividades profesionales de cada uno de los ámbitos profesionales referidos. Por último, los aspectos por evaluar identifican los conocimientos y habilidades necesarios para realizar tareas específicas relacionadas con cada actividad profesional (tabla 3 ).

En el examen se utilizan preguntas de opción múltiple que contienen fundamentalmente los siguientes dos elementos:

- La base es una pregunta, afirmación, enunciado o gráfico acompañado de una instrucción que plantea un problema explícitamente.

- Las opciones de respuesta son enunciados, palabras, cifras o combinaciones de números y letras que guardan relación con la base de la pregunta, donde sólo una opción es la correcta. Para todas las preguntas del examen siempre se presentarán cuatro opciones de respuesta (tabla 4).

\section{ESTADOS UNIDOS}

El propósito del examen The Registration Examination for Dietitians (Examen de Registración para los Dietistas) (8) es de proteger la salud del público mediante la determinación de la competencia del individuo para practicar la profesión. El examen es un test interactivo y computarizado administrado por el American College Testing (ACT), una empresa que también administra exámenes para otras profesiones. El examen es de formato de opción múltiple (llamado "ítem" por el ACT). Es un test computarizado adaptativo, porque el software tiene reglas de selección de ítems y reglas para detener el test. Hay dos tipos de ítems (conocimiento y aplicación).

El contenido del examen está basado en una auditoria de la práctica profesional de Dietistas Registrados (RD) entrando a la profesión en los EEUU, tiene una validez de cinco años y es administrado por el Center Dietetics Registration (CDR). Las especificaciones del examen del CDR y los resultados de aprendizaje de los estudiantes requeridos por la Commission Acreditation Dietitcs Evaluation (CADE) no son exactamente iguales,

\section{TABLA 4}

\section{Niveles de Desempeño}

El EGEL-NUTRI permite identificar el nivel de dominio o desempeño logrado por el egresado con respecto a los conocimientos y habilidades que el Consejo Técnico del Examen ha definido como necesarios para iniciarse eficazmente en el ejercicio profesional. Cuando un egresado obtiene niveles satisfactorio (2) y sobresaliente (3) en el examen, implica que ha demostrado contar con los conocimientos y habilidades que están siendo evaluados. A continuación se describe cada uno de esos dos niveles.*

Nivel de desempeño satisfactorio (2)

Atención clínica nutricional. El egresado atiende las necesidades nutricionales del individuo por lo que atañe a evaluación, tratamiento, orientación y administración del servicio de nutrición clínica.

Atención nutricional a grupos de individuos. El egresado reconoce los elementos básicos de una situación alimentaria y nutricional de un grupo poblacional, además de los principios básicos de intervenciones y de la construcción de protocolos de investigación aplicados a la nutrición

Administración de los servicios de alimentos. El egresado identifica los elementos implicados en el manejo del servicio de alimentos, la normatividad elemental, el empleo de planes alimentarios y menús y las características de la orientación alimentaria.

\section{Nivel de desempeño sobresaliente (3)}

Atención clínica nutriológica. El egresado reconoce los procedimientos y resuelve los problemas relacionados con la evaluación, tratamiento, orientación y administración de los servicios de nutrición clínica de acuerdo con las patologías y necesidades de los individuos.

Atención nutriológica a grupos de individuos. El egresado aplica criterios estructurados para la resolución de casos específicos en situaciones alimentarias y nutricionales de la población. Es capaz de discernir -entre diagnósticos, políticas públicas, estrategias de solución y protocolos de investigación- las mejores alternativas para la atención a los grupos poblacionales.

Administración de los servicios de alimentos. El egresado ofrece alternativas de mejora continua en el manejo de los servicios de alimentos, acatando la normatividad y la orientación alimentaria y empleando diversas técnicas en el diseño de planes alimentarios y menús. 
así que los educadores de la dietética deben estar atentos a ambos en una preparación óptima de sus estudiantes para el éxito en el examen (9-10).

Las etapas son la redacción de ítems, la revisión de ítems y el test preliminar (pre-test pre-testing) de ítems. Lo pueden dar en cualquier de las 200 ubicaciones a través de los EEUU con cita previa en un centro de examen.

El examen consiste en 105 a 125 ítems con puntaje, más 20 ítems en prueba (sin puntaje). El software selecciona ítems basados en su categoría de contenido, dificultad y tipo (conocimiento o aplicación). Hay tres escenarios de examen. En el primero, si le va muy bien al candidato en la mayoría de las preguntas del examen en todas las áreas de contenido, el examen se detendrá después de 105 ítems con puntaje, porque el estudiante ha aprobado el examen. No hay necesidad para el candidato de seguir contestando más preguntas para que el software haga una decisión aprobar-reprobar. En el segundo caso, si el candidato dio respuestas incorrectas en muchas preguntas, así que llegando a 105 ítems con un puntaje sin ninguna posibilidad de aprobar el examen se detendrá y el candidato reprobará. En el tercer escenario, si el candidato respondió correctamente a todos los ítems de dificultad más fácil en una categoría dada pero incorrectamente en los ítems de mayor dificultad. En este caso, el software seguirá ofreciendo ítems hasta recolectar los datos suficientes para tomar una decisión aprobar-reprobar (11).

La tasa de aprobación general por todos los tipos de programas ha sido estable por años en aproximadamente $80 \%$ de las personas que rinden el examen.

El examen dura 3 horas, media hora tutorial para familiarizarse con el examen y $2 \square$ hora para rendir el examen (tabla 5).

\section{CANADÁ}

La alianza del cuerpo regulatorio de la Dietética en Canadá se encarga de mantener un estándar uniforme para entrar a la profesión de la Dietética, llamado Dietista la que tiene sus cualificaciones reconocidas por los

TABLA 5

Esquema de estudio (para el examen de registración de dietistas).

DOMINIO I - ALIMENTACIÓN Y NUTRICIÓN (12\%)12

TEMA A - Ciencia de la Alimentación y Composición de los Alimentos

TEMA B - Nutrición y Ciencias de Apoyo

\author{
DOMINIO II - PROCESO Y MODELO DE LA ATENCIÓN DE NUTRICIÓN - \\ CONDICIONES COMPLEJAS (40\%) \\ TEMA A - Tamizaje y Valoración de Nutrición \\ TEMA B - Diagnostico de Nutrición \\ TEMA C - Intervención de Nutrición \\ TEMA D - Monitoreo y Evaluación de Nutrición
}

DOMINIO III - CONSEJERÍA, COMUNICACIÓN, EDUCACIÓN E INVESTIGACIÓN (10\%)

TEMA A - Valoración y Planificación

TEMA B - implementación y evaluación

TEMA C - investigación

DOMINIO IV - SISTEMAS DE ALIMENTACIÓN Y NUTRICIÓN (17\%)

TEMA A - Planificación de Menú

TEMA B - Adquisiciones, Producción, Distribución y Servicio

TEMA C - Seguridad y Sanidad

TEMA C - planificación de las instalaciones

DOMINIO V - GESTIÓN (21\%)

TEMA A - Recursos Humanos

TEMA B - Finanza y Materiales

TEMA C - Marketing de Productos y Servicios

TEMA D - Funciones y Características

TEMA E - Mejoramiento de la Calidad 


\section{TABLA 6}

\section{Hechos: El examen}

- Se apoya sobre los diverso criterios de evaluación

- Refleja el ejercicio actual de la dietética en Canadá

- Está fundada sobre las competencias

- No es un test de diagnóstico de competencias

- Los resultados se expresan como aprobado o desaprobado

- Está revisado atentamente y varias veces por diferentes personas

- No genera ninguna ganancia (lo que explica el costo de inscripción)

- Formato del examen y de las preguntas

\section{Explicaciones}

- Permite comparar los candidatos desde un criterio único, es decir las competencias mínimas.

- Se distingue así de la mayoría de los exámenes de tipo normativo que comparan el rendimiento de los candidatos desde una nota de aprobación fijada arbitrariamente.

- Permite evaluar seis categorías de competencias: el ejercicio profesional, valoración, planificación, implementación, evaluación y comunicación.

- Estas categorías han sido definidas y validadas al nivel nacional en 1995 por la asociación canadiense de Dietistas (ahora los DC).

- Ha sido concebido con el propósito de verificar si el egresado. Tiene las competencias mínimas requeridas

- No ha sido concebido para medir el nivel de competencias

- Los resultados son indicados por la mención aprobado (adquisición de las competencias mínimas requeridas) o desaprobado (no adquisición de las mínimas requeridas).

- En el caso de desaprobación no recibirán nada más que la mención desaprobado.

- Si ha desaprobado, una revisión completa de las competencias se impone

- Una empresa especializada en preparación de exámenes cuya experiencia es reconocida está contratada para gestionar el examen.

- El comité de examen, redactores de ítem, evaluadores de ítem y el comité de revisión de traducción en francés juntan dietistas profesionales de todas las regiones del país que tiene experiencia reconocida en diversas áreas de la profesión o al nivel de la enseñanza universitaria o de la formación práctica. Estas personas actúan como redactores o evaluadores de las preguntas.

- Cada pregunta se revisa al menos 10 veces para asegurar que el examen:

- Evalúa las competencias del nivel de entrada a la profesión

- Refleja adecuadamente la situación actual del ejercicio de la dietética a nivel nacional

- El examen se toma en dos sesiones de 3 horas (mañana y tarde) 
Hechos: El examen

Dominio cognitivo

- Cada pregunta evalúa uno de los tres niveles de capacidad

Clasificación de las competencias

- Cada pregunta enfoca una competencia y no un dominio de practica

- Algunas competencias más importantes son evaluadas con más frecuencia que otras

Variables contextuales

- Edad/sexo del cliente

- Cultura

- Entorno de atención de salud

\section{Explicaciones}

- Se puede salir del examen una hora $1 / 2$ después de que empiece cada sesión

- El examen tiene de 200 a 220 preguntas de selección múltiple

- 20 a 30\% de las preguntas están basadas en casos (3 a 6 preguntas por cada caso)

- $70-80 \%$ de las preguntas son independientes

- 20 - 30\% de las preguntas evalúan los conocimientos/la comprensión (capacidad de memorizar/comprender los conocimientos adquiridos)

- 45 a 55\% de las preguntas evalúan la aplicación (capacidad de aplicar sus conocimientos en nuevas situaciones concretas)

- 20 - 30\% de las preguntas evalúan el pensamiento crítico (capacidad de juzgar la pertinencia de datos, manejar abstracciones y resolver problemas).

- Cada pregunta evalúa una competencia

- Se utilizan diversos contextos para representar todos los dominios de la práctica.

- Por ejemplo una pregunta puede ser sobre la planificación o la evaluación sin ser una pregunta clínica o administrativa.

- Los candidatos frecuentemente enfocan en la información sobre la situación más que en la pregunta de fondo que siempre evalúa una competencia; esta es la idea falsa más persistente sobre el examen.

- Estos se refleja en la proporción de preguntas en el examen relacionadas a cada grupo de competencias: - Grupo 1A Competencias muy importantes frecuencia alta $(40-50 \%)$

- Grupo 1B competencias muy importantes baja frecuencia (30-40\%)

- Grupo 2A Competencias importantes frecuencia alta $(15-25 \%)$

- Grupo 2B Competencias importantes baja frecuencia $(1-10 \%)$

- Las preguntas proveen una variedad de contextos que reflejan la práctica al nivel de entrada a la profesión en Canadá

- Los temas culturales no están basados sobre ningún estereotipo. Todas las preguntas han sido formuladas desde la lista de temas de referencia. 
empleadores de Canadá. Los miembros de la Alianza seleccionan requerimientos comunes por la academia y el entrenamiento practico, las competencias para entrar a la profesión, los estándares de la profesión, el ámbito de la práctica profesional y el código de ética.

El cuerpo regulatorio (universidades, asociaciones y colegios profesionales) protege al público asegurando que los dietistas están educados y capacitados, de esta forma mantienen la alta calidad de atención en nutrición en Canadá. Canadian Dietetic Registration Examination (CDRE) (13) no es un examen de salida del internado o de un programa de capacitación práctica, sino que está diseñado para en confirmar la práctica basada en el conocimiento, aplicación y habilidades en pensamiento crítico, está a un nivel mínimo de competencias que el dietista estará seguro de practicar.

El examen es la etapa final en proceso de registración para llegar a ser un Dietista Registrado (RD) y tiene como único propósito de distinguir entre practicantes competentes y no competentes. Tiene una validez de 5 años, en los cuales los RD deben demostrar haberse actualizado mediante cursos, asistencia a congresos,

\section{TABLA 7}

\section{Como leer las preguntas del examen}

\section{Etapa 1}

Leer el texto de la pregunta para determinar

a) El nivel cognitivo

b) La categoría de la competencia

\section{Etapa 2}

Leer de nuevo el texto además de la selección de respuestas.

\section{Etapa 3}

Elige la respuesta correcta dentro de la selección presentada.
- Evite de clasificar la pregunta en categorías como diabetes, nutrición clínica, etc. Las explicaciones describen el entorno en el cual se evalúa una competencia

- Intente identificar el nivel cognitivo de la pregunta, se le pide

- Informaciones (conocimientos/comprensión)

- Aplicación de informaciones (Aplicación)

- Análisis de informaciones (pensamiento crítico)

- Relacione la pregunta a una de las 6 categorías de competencias

Se le pide demostrar sus competencias en:

Ejercicio profesional, implementación, valoración, evaluación, planificación o comunicación.

- Determine si la pregunta es un aspecto temporal (un punto en el tiempo). Es decir se le pide un paso inicial en un proceso o un paso final, etc.

- Recuerde que el examen no tiene pregunta trampa

- Las respuestas falsas (o distractoras) sirven para revelar un nivel insuficiente de conocimientos, de aplicación o de pensamiento crítico.

- Si Ud. Cree que falta información hay que releer la pregunta más atentamente (etapa 1)

- Todas las informaciones requeridas para contestar correctamente las preguntas están dadas.

- Todas las informaciones no pertinentes están excluidas para evitar inducir al error y hacer perder tiempo. 
publicaciones etc.

Se fundamenta en hechos y explicaciones que se muestran en la tabla 6 .

Algunas instrucciones que se dan para un mejor rendimiento en el examen se muestran en la tabla 7 , destacando 3 etapas de como leer las preguntas.

\section{CONCLUSIONES}

Existen diversos tipos de exámenes para medir las competencias del nutricionista que ingresa a la profesión, sin embargo todos ellos tienen el mismo objetivo como lo expresa el ENGEL-NUTR, sirve para que el egresado mida sus competencias, las instituciones formadoras evalúen su calidad y a los empleadores sea una herramienta de selección. El examen en Canadá y Estados Unidos tiene una validez de 5 años durante los cuales las Dietistas Registradas deben demostrar haberse actualizado, lo mismo ocurre para la certificación de las especialidades.

Los cuatro exámenes mostrados son diversos en la metodología de aplicación, por lo que es importante estudiar de manera muy profunda cual será la metodología que podría aplicarse en Chile, pensando en lo que se quiere, es que el egresado demuestre las competencias para ejercer la profesión, por ello se duda si la aplicación de un examen con preguntas de selección múltiple con una respuesta correcta como lo son el de Colombia o México, logran demostrar competencias, al parecer un método como el de Estados Unidos es más efectivo.

En Chile dado la gran cantidad de Instituciones que ofrecen la carrera se hace indispensable su aplicación, el que debiera estar a cargo de una institución autónoma dependiente del Ministerio de Educación o Salud, con la representación del Colegio de Nutricionistas Universitarias de Chile y académicos de carreras de Nutrición y Dietética Acreditadas. Previo a la aplicación del examen es necesario, establecer las competencias profesionales y de estas, determinar las competencias de un egresado de nutrición que se inicia en el trabajo profesional, las que servirían de base para todas las universidades que ofrecen la carrera de Nutrición y Dietética unificando la formación del nutricionista. Estas competencias deberían ser conocidas por los estudiantes, académicos y empleadores y podrían ser la base para la acreditación de la carrera, utilizando estándares específicos de la profesión.

Por otro lado, a fin de asegurar la calidad de la atención en las diversas patologías AUGE como diabetes mellitus, insuficiencia renal, obesidad, hipertensión, entre otras, el Ministerio de Salud está solicitando la certificación de las especialidades en Nutrición y Dietética, para ello se debería establecer las competencias del nutricionista por especialidad.

\section{RESUMEN}

En Chile actualmente hay 68 opciones que ofrecen la carrera de Nutrición y Dietética, solo 6 programas están acreditados. Por ello, resulta indispensable aplicar un examen que mida las competencias mínimas para entrar a ejercer la profesión. Se estudió la aplicación del examen en cuatro países. En Colombia el propósito es indagar el perfil de competencias cognoscitivas, al identificar información relevante, interpretar situaciones, argumentar decisiones y proponer alternativas de acción profesional en cada una de los componentes de la nutrición. En México es identificar si los egresados cuentan con los conocimientos y habilidades necesarios para iniciarse eficazmente en el ejercicio de la profesión. En Canadá se ha diseñado para confirmar la práctica basada en el conocimiento, aplicación y habilidades en pensamiento crítico, está a un nivel mínimo de competencias que el dietista deberá practicar, tiene como único propósito de distinguir entre practicantes competentes y no competentes. En Estados Unidos la finalidad del examen es de proteger la salud del público mediante la determinación de la competencia del individuo para practicar la profesión, es un test interactivo y computarizado. Se concluye que la aplicación de un examen nacional para ejercer la profesión de Nutricionista debería aplicarse lo antes posible en Chile.

Palabras clave: examen nacional, nutrición, competencias, conocimientos y habilidades.

Dirigir la correspondencia a:

Profesora

María Angélica González S.

Nutricionista MSc.

Dpto. Nutrición y Salud Pública

Fac. Cs. de la Salud y de los Alimentos

Universidad del Bio-Bío, Chile

Fono: 42 - 253121 42-253076

Fax: $42-253142$

E-mail:magonzal@ubiobio.cl

\section{BIBLIOGRAFÍA}

1. Consejo Nacional de Educación. Estadísticas y bases de datos. http://www.cned.cl/public/Secciones/SeccionIndicesEstadisticas/estadisticas_Buscar_programas_resultado.aspx?sch $=1 \&$ bitPro $=1 \&$ area_9=9\&nombrePrograma=Nutricion y dietética. Consultado 26/07/2011.

2. Colegio de Nutricionista Universitarios de Chile. http://www.nutricionistasdechile.cl/estudios.html. Consultado 26/07/2011. 
3. Consejo Nacional de Educación. Estadísticas bases de datos (2009-2010-2011). www.cned.cl. Consultado 26/07/2011.

4. Consejo Nacional de Educación. Estadísticas y bases de datos http://www.cned.cl/public/ Secciones/SeccionIndicesEstadisticas/estadisticas_comparar_programas_resultado.aspx?criterio $=8 \&$ bitarribaabajo=1\&tipoIP_1=1\&bitPro=1\&bit Tec $=0 \&$ bitBac $=0 \&$ nombrePrograma $=$ Nutricion $y$ Dietetica\&intAnio=2010. Consultado 26/07/2001

5. Cruces M. Richard. Evolución de la Formación de Recurso Humano Nutricionista Universitario. Rev Colegio Nutricionistas 2007;7(1):20-1.

6. Exámenes de calidad de la educación superior en nutrición y dietética. Guía de Orientación. Bogotá D. Instituto Colombiano Para El Fomento De La Educación Superior. ICFES. ISSN: 1794 - 595X. 2009.

7. Guía para el sustentante. Examen General para el Egreso de la Licenciatura en Nutrición. EGELNUTRI Nueva Generación. Centro Nacional de Evaluación para la Educación Superior, A.C. CE-
NEVAL. 2010. Cuarta edición.

8. Innovaciones académicas para optimizar la educación y formación de nutricionistas Proyecto MECESUP. Informe de la Profesora en visita de experta Kay Stearns Bruening. 14 al 26 de Noviemhre del 2010

9. htm:/www.testprepreview.com/modules/dietetics htm consultado 9/08/2011.

10. Study Guide for the Registration Examination for Dietitians, 9th Ed. American Dietetics Association

11. ADA. Commission on Dietetics Registration. Registration Examination for Dietitians Handhook for Candidates 2011 http./www cdrnetorg/pdfs/ ADA\%20CDR\%20RD\%20Handbook.pdf Consultado el 9/08/2011.

12. Commission on Dietetic Registration, Registration Examination for Dietitians Study Outline STUDY OUTLINE Ww Study\%20Outline-Syllbus\%209-06.pdf. Consultado el 9/08/20011.

13. Canadian Dietetic Registration Examination (CDRE). Preparation Guide. 2011. 